\title{
9. Affinrotationshyperfä̈chen.
}

Von Wilhelm Süss in Kagoshima.

Herrn Professor Kosaburo Ishikura in Dankbarkeit gewidmet.

(Eingegangen am 27. Februar, 1928.)

\section{Einleitung.}

1. In einer in den Mathematischen Annalen* erscheinenden Arbeit habe ich ein affingeometrisches Gegenstück der elementaren Rotationsflächen betrachtet: diejenigen Flächen, deren Affinnormalen sämtlich eine feste Gerade a, die "Achse ", treffen und deren "Meridiane" Schattengrenzen sind. Unter den Meridianen sind dabei die Schnittkurven der Fläche mit den durch die Achse gelegten Ebenen zu verstehen. Die Affinrotationsflächen $(A-R-F)$ sind dadurch ausgezeichnet, dass durch jeden ihrer Punkte zwei reelle Affinkrümmungslinien gehen, diese sämtlich ebene Kurven sind und ferner diejenigen der einen Schar, die „Breitenkurven“, in parallelen Ebenen verlaufende, einander ähnliche und zur Achse $a$ ähnlich gelegene Kegelschnitte sind. Die $A-R-F$ gehören übrigens, wie hier beiläufig bemerkt sei, zu den „Projektiv-Rotationsflächen ", welche durch analoge Merkmale charakterisiert sind und ähnliche Eigenschaften aufweisen, worauf ich bei anderer Gelegenheit einzugehen gedenke. Hier sollen die früheren Ergebnisse auf Hyperflächen im $(n+1)$-dimensionalen Raum ausgedehnt werden. Dabei wird sich zugleich eine im Falle $n=2$ übersichtlichere Beweisanordnung ergeben.

2. Wir unterscheiden "eigentliche" und „uneigentliche" Affinrotationshyperflächen (A-R-H), je nachdem ob die Achse $a$ im Endlichen liegende Punkte enthält oder nicht. Eigentliche $A-R-H$ sind nach der Bezeichnungsweise der Vorlesungen über Differentialgeometrie ron W. Blaschke( $\left.{ }^{1}\right)$, der wir uns hier anschliessen, durch die Gleichung

$$
\mathfrak{x}+\alpha \mathfrak{y}=\beta_{\mathfrak{a}}
$$

(") Band 98

(1) Band II, Berlin 1923; dieses Buch wird mit B, die Formeln des \$ 6j werden ohne Seitenangabe zitiert. 
definiert, worin $\alpha$ und $\beta$ Ortsfunktionen auf der $A-R-H$ sind, die wir der Einfachheit wegen wie $x$ als analytisch voraussetzen, während a der feste Einheitsvektor der Achse a sein soll. Der Ursprung $O$ liegt dabei auf $a$.

Bei den uneigentlichen A-R-H sind alle Affinnormalen zu einer festen (zweidimensionalen) Ebene $\eta$ parallel. Es gibt also $(n-1)$ wechselseitig orthogonale Einheitsvektoren $\mathfrak{z}^{(i)}$, auf welchen $\mathfrak{y}$ senkrecht steht:

$$
y^{(i)}=0, j^{(i ; 2}=1, j^{(b)} 3^{(k)}=0, i \neq k i, k=1,2, \ldots,(n-1)
$$

wodurch diese $A-P-H$ definiert werden.

Wir setzen noch voraus, dass die Determinante der quadratischen Grundform nicht verschwindet,

$$
G=G_{i n} \neq=0 \quad(B(195)),
$$

wodurch wir Torsen ausschliessen.

Ich behaupte: Die A-R-H haben stets n linear unabhängige reelle Afinkrïmmungstinien. Thre Meridiane sind Affnkrïmmungstinien; ithe "Breitenmannigfaltigkeiten", fül" die die Affinnormalen der A-R-H alle durch denselben Punkt gehen, sind $(n-1)$-dimensionale, in parallelen n-dimensionalen Ebenen gelegene, einander ähnliche und zur Achse a änlich gelegene Affinhypersphären; diese sind eigentlich oder uneigentlich, je nachdem ob die A-R-H eigentlich oder uneigentlich ist.

Den Affinsphären, zu denen bekanntlich u. a. die Flächen zweiter Ordnung gehören, entsprechen im Falle $n=1$ die Kegelschnitte, sodass der soeben ausgesprochene Satz den in den Math. Annalen reröffentlichten als Sonderfall umfasst.

3. A-R-H haben in jedem Punkt n linear unabhängige Affnkrümmungstinienvichtungen. Denn nach (1) sowohl wie nach (1) gibt es zu jedem Punkt der $A-P-H$ eine $(n-1)$-dimensionale Nannigfaltigkeit von Fortschreitungsrichtungen, für welche die Funktion $\beta$ ihren Wert beibehält; jede dieser Richtungen stellt eine solche einer Affinkrümmungslinie nach deren Definition dar. Sehen wir nun von Affinhypersphären ab, für welche die Behauptung von vornherein gilt, so lässt sich eine Affinkrümmungslinie angeben, die nicht $z u$ den vorigen gehört. Jede zweidimensionale Ebene durch die Achse a schneidet nämlich die A- $R-H$ nach deren. Definition in einer Affinkrümmungslinie, die wir "Meridian" nennen. Nach unserer anfangs gemachten Bemer" kung befinden sich unter den zu den Meridianen und einander wechselseitig konjugierten Kurven, den "Breitenkurven", mindestens 
$(n-2)$ Affinkrümmungslinien. Wir betrachten zuerst nur eigentliche $A-R$ H. Die Richtungen aller dieser $(n-1)$ Affinkrümmungslinien, deren Affinnormalen die Achse in einem und demselben Punkte $S$ treffen, spanne in einem Punkt $P$ der $R-A-H$ eine $(n-1)$-dimensionale Ebene $E_{n-1}$ auf. Gehen wir von einer Richtung des Meridians durch $P$ ans und bewegen sie stetig in der $n$-dimensionalen Tangentenebene $E_{n}$ von $P$ bis in die zur Ausgangslage entgengesetzte Richtung, so muss, da wir Affinhypersphären ausgeschaltet haben, die Affinnormale die Achse $a$ in von $S$ verschiedenen Punkten treffen, wenn wir den bewegten. Richtungsvektor nur so in $E_{n}$ führen, dass er mit Ausnahme seiner Ausgangs- and Endlage nicht in die $E_{n-1}$ fällt. Der gleichzeitig bewegte Schnittpunkt der Affinnormalen kehrt schliesslich in den Punkt $S$ zurück, muss also während des Bewegungsvorganges eine Umkehrlage seiner Bewegung auf $a$ einnehmen. Die dieser Umkehrlage zugeordnete Richtung von $P$ aus in $E_{n}$ ist aber dann eine weitere Affinkrümmungslinienrichtung, w. z. b. w. Wie man erkennt, ist der Wortlaut unserer Argumentation im Falle uneigentlicher A-R-H nur wenig zu ändern, um zum gleichen Ziel zu gelangen.

4. Wählen wir die Affinkrümmungslinien zu Parameterkurven, so ist

$$
G_{i k}=B_{i k}=C_{i k}=0 \text { für: } i \neq k(i, k=1,2, \ldots \ldots, n),
$$

da sie ein konjugiertes System bilden, da ferner die Formeln von $O$. Rodrigues längs ihnen gelten

$$
\mathfrak{x}_{i}+R_{i} \mathfrak{y}_{i}=0
$$

and da drittens die Beziehung

$$
C_{i k}=A_{i k i, l}^{l}=\frac{n}{1}\left[H G_{i k}+B_{i k}\right]
$$

gilt $\left({ }^{2}\right)$. Wir wollen noch unsere definierende Bedingung der $A-R-H$ analytisch fassen, dass die Meridiane, die $u^{1}$-Parameterlinien $\left(u^{k}=\right.$ const für $k>1$ ), Schattengrenzen für die Richtungen der Breitenkurven, der $u^{k}$-Parameterlinien, $(k>1)$, sein sollen. Diese Bedingung lautet

$$
\frac{\partial \mathfrak{r}_{k}}{\partial u^{1}} \times x_{k}=0
$$

oder wegen der Ableitungsgleichungen $B$ (200)

$\left({ }^{2}\right)$ Vergl. ansser B noch I. Bepracld: Monatshefte für. Math. 32 (1922) 89-106. 


$$
\begin{aligned}
A_{1 k^{b}}+\Gamma_{1 k}^{l}=0 \quad(k \neq 1, k & =2,3, \ldots \ldots, n, \\
l & =1,2, \ldots \ldots, n) .
\end{aligned}
$$

Hieraus folgt noch wegen. (2)

$$
A_{1 k m}=\Gamma_{1 k m}=0 \quad(k \neq m ; k, m=2,3, \ldots \ldots, n) .
$$

\section{Eigentliche A-R-H}

5. Für eigentliche $A-R-H$ folgt aus (1) und (2)

$$
\left(1-\frac{\alpha}{R_{i}}\right) \mathfrak{x}_{i}+\alpha_{i} \mathfrak{\eta}_{i}=\beta_{i} \mathfrak{a} \quad(i=1,2, \ldots \ldots, n) .
$$

Für $i \neq k$ kann die hieraus folgende Gleichung

$$
\beta_{k}\left(1-\frac{\alpha}{R_{i}}\right) \mathfrak{x}_{i}-p_{i}\left(1-\frac{\alpha}{R_{k}}\right) \mathfrak{x}_{k}+\left(\alpha_{i} \beta_{k}-\alpha_{k} \beta_{i}\right) \mathfrak{y}=0
$$

wegen der linearen Unabhängigkeit der Vektoren $x_{1}, x_{2}, \ldots, x_{n}, \mathfrak{n}(G \neq 0$ in Nr. 2) auf drei Arten erfüllt werden:

A)

$$
\begin{aligned}
& \beta_{i}=\beta_{k}=0, \quad \operatorname{also} \operatorname{nach}(4) \\
& \alpha_{i}=\alpha_{k}=0, \quad R_{i}=R_{k}=\alpha ;
\end{aligned}
$$

B) $\quad R_{i}=R_{k}=\alpha, \alpha_{i} \beta_{k}=\alpha_{k} \beta_{i}$; hier ist nach (4) umgekehrt $\alpha_{i}=\beta_{i}=\alpha_{k}=\beta_{k}=0$, die Fälle A) und B) sind also miteinander identisch.

C) $\alpha_{k}=\beta_{k}=0, \alpha=R_{k}$. Der Fall $C$ ) ist nur dann von A) und B) verschieden, wenn wir ausdrücklich $\beta_{i} \neq 0$ annehmen.

Ist für alle $i \quad \beta_{i}=0$, so sind nach $A$ ) alle Affinkrümmungsradien einander und $\alpha$ gleich; dann ist die $A-R-H$ zugleich eine (eigentliche) Affinhypersphäre. Andernfalls ist für einen einzigen Wert $i$, etwa $i=l$, $\beta_{l} \neq 0$, während $\beta_{i}$ für alle andern $i$ verschwindet. Ich behaupte, dass in diesem Falle $l=1$ sein muss; denn wäre $l \neq 1$ und also nach $C$ ) $\alpha_{l} \neq 0, \beta_{l} \neq 0$, aber $\alpha_{1}=\beta_{1}=0, \alpha=R_{1}$, so folgte aus (4)

$$
\frac{\partial \mathfrak{x}_{l}}{\partial u^{1}}\left(1-\frac{\alpha}{R_{l}}\right)-\alpha\left(\frac{1}{R_{l}}\right)_{1} \mathfrak{x}_{l}+\alpha \mathfrak{y}_{1}=0
$$

nach (3) wäre also $\alpha_{l} \mathfrak{y}_{1}=0$, also $\mathfrak{y}_{1}=0$, da wir $\alpha_{l} \neq 0$ angenommen hatten, d. h. also $\frac{1}{R_{1}}=0$, was indessen wegen der Bedeutung der Funktion $\alpha=R_{1}$ in (1) ausgeschlossen ist. Wir können also nach A), B), C) setzen : 


$$
\begin{array}{ll}
\alpha_{1} \neq 0, \beta_{1} \neq 0, & R_{1} \neq R_{k} \\
\alpha_{k}=\beta_{k}=0, & R_{k}=\alpha
\end{array}
$$

Da nach (1) und (4)

$$
\mathfrak{x}+\frac{\alpha}{\alpha_{1}}\left(\frac{\alpha}{R_{1}}-1\right) \mathfrak{x}_{1}=\left(\beta-\frac{\alpha \beta_{1}}{\alpha_{1}}\right) \mathfrak{a}
$$

ist, so folgt aus (5), dass alle Tangenten an die Meridiane die Achse a längs einer Breitenmannigfaltigkeit $u^{1}=$ const in einem und demselben. Punkte treffen.

Nach (2), (4) und (5) ist nun

$$
\mathfrak{a} X_{k}=(\mathfrak{a} X)_{k}=0 \quad(k>1),
$$

wegen (1) also auch

$$
\mathfrak{r} X_{k}=(\mathfrak{x} X)_{k}=0 \quad(k>1),
$$

d. h. die Affinentfernung der Punkte einer Breitenkurve von einem Punkt der Achse a ist konstant. Dies ist ein neues Gegenstück zu den Verhältnissen bei den gewöhnlichen Rotationsfächen; ein weiteres besteht in den Gleichungen

$$
\frac{\partial R_{1}}{c u^{k}}=0 \quad(k>1)
$$

aus (4) folgt nämlich

$$
\frac{\partial \mathfrak{x}_{1}}{\partial u_{i}^{k}}\left(1-\frac{\alpha}{R_{1}}\right)-\alpha \mathfrak{x}_{1}\left(-\frac{1}{R_{1}}\right)_{k}+\alpha_{1} \mathfrak{y}_{k}=0,
$$

woraus sich infolge (3) die Behauptung (8) ergibt.

6. Die Meridiane waren nach Nr. 3 in Ebenen $\eta_{1}\left(u^{2}, u^{3}, \ldots \ldots, u^{n}\right)$ gelegene Affinkrümmungslinien; wir zeigen jetzt, dass die Breitenkurven in den n-dimensionalen Ebenen $\mathrm{D}\left(u^{1}\right)$ verlaufen, in velchen sie für einen bestimmten Punkt beginnen.

Wir beginnen mit einigen rechnerischen Torbereitungen. Die Ebenen $\eta_{1}$ werden von je zweien der Vektoren $a, \mathfrak{b}, \mathfrak{x}, x_{1}, \ldots .$. aufgespannt. Es ist also wegen

$$
\begin{gathered}
\left(\mathfrak{x}_{1}, \frac{\partial \mathfrak{x}_{1}}{\partial u^{1}}, \mathfrak{l}\right)=0: \\
A_{11}^{k}+\Gamma_{\mathrm{l} 1}^{k}=0 \quad(k>1),
\end{gathered}
$$

woraus wegen $(2),(3)$ und 


$$
\Gamma_{i k, l}+\Gamma_{i l, l}=\frac{\partial G_{k l}}{\partial u_{b}^{i}}
$$

folgt:

$$
A_{11 k}=A_{11}^{k}=A_{1 k}^{1}=\Gamma_{11}^{k}=\Gamma_{1 k}^{1}=\frac{\partial G_{11}}{\partial u^{k}}=0 \quad(k>1)
$$

Hiemach ist es erlaubt anzunehmen, der Parameter $u^{1}$ sei so bestimmt, dass

$$
G_{11}= \pm 1, \quad \Gamma_{11}^{1}=0 \text { ist. }
$$

Unsre Behauptung fur die Breitenkurven lantet

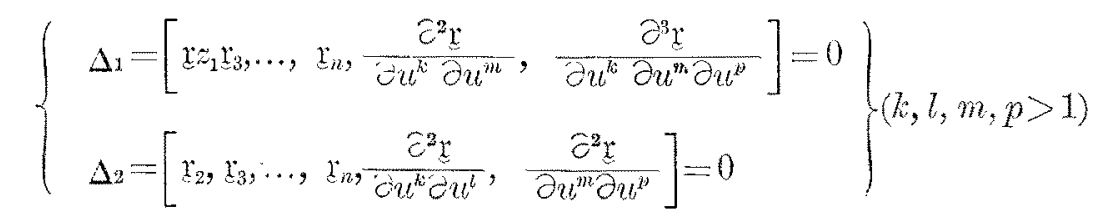

Wir beweisen zunächst (11) . Wegen (2) und $\left(3^{7}\right)$ ist $(11)_{1}$ für $k \neq m$ stets erfült. Ist in (11) fermer $k=m \neq p$, so folgt nach (2) und $\left(3^{b}\right)$

$$
\begin{aligned}
& \frac{\partial^{3} v}{\left(\partial u^{k}\right)^{2} \partial u^{p}}=\frac{\partial}{\partial u^{k}}\left[\left(A_{k p}^{s}+\Gamma_{k k_{p}}^{s}\right) r_{s}\right]=\left(A_{k p}^{s}+\Gamma_{k p}^{s}\right)_{k,} r_{s} \\
& \left.+\left(A_{k i p}^{s}+\Gamma_{k i p}^{s}\right)\left[\left(A_{s: i}^{r}+\Gamma_{s k}^{v}\right)_{z r}+G_{s h}^{\gamma} \mathfrak{l}\right)\right]
\end{aligned}
$$

hierin verschwinden nach $\left(3^{h}\right)$ alle Glieder mit $s=1$ und es wird

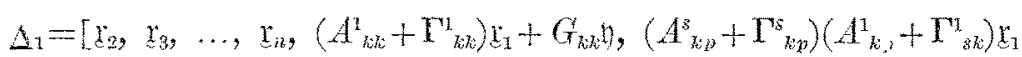

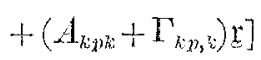

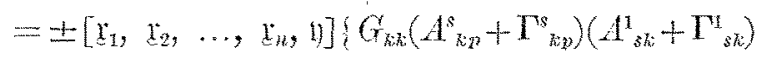

$$
\begin{aligned}
& \left.-\left(A_{k k i}^{1}+\Gamma_{1 k k}^{\prime}\right)\left(A_{k k_{j}}+\Gamma_{k, k_{k}}\right)\right\}=0 \text {, }
\end{aligned}
$$

da hierin in der Summe nach $\left(S^{\prime}\right)$ nur das Glied mit $s=k$ nicht verschwindet. Es bleibt also weiterhin nur noch (11) im Falle $k=m=p$ zu beweisen. Dafur wird

$$
\begin{aligned}
& \Delta_{1}=\left[\mathfrak{r}_{2}, \mathfrak{x}_{3}, \ldots, \mathfrak{x}_{n},\left(A_{k k i}^{\mathrm{I}}+\Gamma_{k k}^{1}\right) \mathfrak{r}_{1}+G_{k k k} \mathfrak{y},\left(A_{k k i}^{1}+\Gamma_{k k}^{1}\right)_{k} x_{1}\right.
\end{aligned}
$$

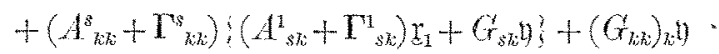

$$
\begin{aligned}
& \left.= \pm\left[\underline{r}_{1}, x_{2}, \ldots, x_{k}, 1\right)\right] ; G_{k k}\left(A_{k k}^{s}+\Gamma_{k k}^{s}\right)\left(A_{s k}^{1}+\Gamma_{s k}^{1}\right)
\end{aligned}
$$

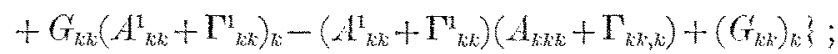


hierin braucht man nach $\left(3^{\prime}\right)$ und $(9)$ nur noch den Wert $s=k$ zu berucksichtigen; es folgt also

$$
\Delta_{1}= \pm\left[\mathfrak{r}_{1}, x_{2}, \ldots, x_{n}, \mathfrak{l}\right]\left\{G_{k k}\left(A_{k k}^{1}+\Gamma_{k k}^{1}\right)_{k}-\left(G_{k k}\right)_{k}\left(A_{k k}^{1}+\Gamma_{k k k}^{1}\right)\right\}
$$

Bei kurzer Umrechnumg zeigt sich, dass (11) jetzt mit der Behauptung

$$
\frac{\partial}{\partial u^{k}}\left(A_{1 k}^{k^{\prime}}-\Gamma_{k}^{\prime \prime}\right)=0 \quad\left(k=k^{\prime}\right)
$$

identisch ist. (Über $k=k^{\prime}$ ist hierin nicht zu summieren!)

Aus (5), (8) und (9) folgt nun zunächst

$$
\begin{aligned}
& \frac{\partial^{2} \mathfrak{X}}{\left(\partial u^{1}\right)^{2}}=-A_{n 1}^{1} X_{1}+B_{n} X, \\
& \frac{\partial^{3} \mathfrak{X}}{\left(\partial u^{1}\right)^{2} \partial u^{k}}=-\left(A_{n}^{1}\right)_{k} X_{1}+a^{r} X_{i} . \quad(r>1),
\end{aligned}
$$

also wegen (6)

$$
\frac{\partial A^{1}{ }^{1}}{\partial u^{k}}=0, \quad(k>1)
$$

Aus $\left(3^{\prime}\right),(4),(5),(8)$ and $(9)$ folgt ferner

$$
\left(1-\frac{\alpha}{R_{1}}\right) \frac{\partial \mathrm{x}_{\mathrm{1}}}{\partial u^{k}}=\frac{\alpha_{1}}{\alpha} \mathrm{r}_{k}=\left(1-\frac{\alpha}{\bar{R}_{1}}\right)\left(\mathcal{A}_{1 k}^{\prime \prime}+\Gamma_{1 k}^{\prime \prime}\right) \mathrm{x}_{k}
$$

und hieraus

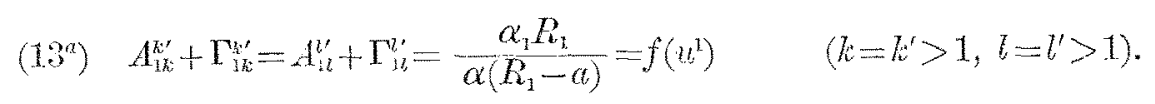

Hieraus folgt wegen der Apolarität und (10)

$$
A_{1 i}^{i}=0=A_{11}^{1}+(n-1) f\left(u^{1}\right)-\Gamma_{1 k}^{i},
$$

nlso nach (12) und (2)

$$
2 \frac{\partial}{\partial u^{k}}\left(\Gamma_{1 i}^{i}\right)=\frac{\hat{c}^{2}(\log G)}{\partial u^{1} \partial u^{k}}=0
$$

Im Falle $n=2$ folgt daraus wegen (12)

$$
\frac{1}{2} \frac{\hat{c}^{2} \log G_{n 1}}{\partial u^{1} \partial u^{k}}=\frac{\partial}{\partial u^{2}}\left(\Gamma_{12}^{2}\right)=0
$$

nach $\left(13^{a}\right)$ also unsere Behauptung $\left(11^{a}\right)$.

Im Falle $n \geq 2$ beachten wir, dass nach den Entwicklungen, die zu (9) geführt haben, für jede dem von $x_{2}, x_{3}, \ldots, x_{n}$ aufgespaunten linearen 
$(n-1)$-dimensionalen Raume angehörende Richtung $x^{\prime}=r_{r}\left(u t^{p}\right)^{\prime}\left(r^{\prime}>1\right)$ die zugehörigen Vektoren $X^{\prime}$ der Bedingung

$$
X^{\prime} \times\left(X_{1}\right)^{\prime}=0 \text { oder }\left(X_{1}\right)^{\prime}=\frac{\partial \mathfrak{K}_{1}}{\partial u^{\prime}}\left(u^{\prime}\right)^{\prime}=B X^{\prime \prime}=B X_{r}\left(u^{\prime}\right)^{\prime} \quad\left(r^{\prime}>1\right)
$$

genügen; da insbesondere

ist, so folgt

$$
\frac{\partial \mathfrak{X}_{1}}{\partial u_{r}^{r}}=\left(-A_{1 r}^{\prime \prime}+\Gamma_{1 r}^{\prime \prime}\right) \ddot{X}_{r}
$$

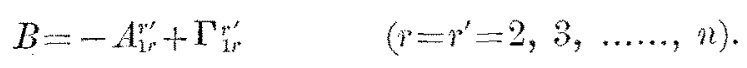

Aus $\left(13^{a}\right)$ und $\left(13^{\circ}\right)$ aber folgt

$$
\Gamma_{1 k}^{k^{\prime}}=\Gamma_{i k}^{\prime \prime}, \quad A_{1 k}^{k^{\prime}}=A_{1 l}^{l^{\prime}} \quad\left(k=k^{\prime}>1, l=l^{\prime}>1\right) .
$$

Nach (12), $\left(13^{*}\right)$ und den Apolaritätsbeziehungen $A^{i}{ }_{1 i}=0$ folgt aber damn die Behauptung $\left(11^{\alpha}\right)$, welche das Terschwinden der Determinante $\Delta_{1}$ besagt. Es ergibt sich darüber hinaus sogar

$$
\frac{\partial}{\partial u^{k}}\left(A_{k}^{k \prime}\right)=\frac{\partial}{\partial u^{k}}\left(\Gamma_{1 k}^{k \prime}\right)=0 \quad(k>1) .
$$

Wir beweisen jetzt $(11)_{2}$ oder, was damit gleichbedeutend ist

$$
G_{k l}\left(A_{m p}^{1}+\Gamma_{m p}^{\mathrm{I}}\right)=G_{m p}\left(A_{k l}^{\mathrm{1}}+\Gamma_{k l}^{1}\right) .
$$

Diese Gleichung ist nach (2) und $\left(3^{k}\right)$ erfüllt, wenn $m \neq p$ oder $k \neq 1$ ist, ausserdem natürlich für $k=1=m=p$. Im Falle $k=1 \neq m=p$ aber folgt sie aus $\left(13_{a}\right)$. Es ist also auch $(11)_{2}$ in allen Fällen als gültig nachgewiesen. Die Mannigfaltigkeiten der Breitenkurven verlaufen also in den linearen n-dimensionalen Räumen $\mathrm{D}\left(u^{1}\right)$, in denen sie in einem ihrer Punkte beginnen. Aus $\left(3^{a}\right)$ folgt noch, dass diese n-dimensionalen Ebenen $\mathrm{D}\left(u^{\mathrm{l}}\right)$ einander parallel sind.

7. Wir untersuchen jetzt eine bestimmte Breitenmannigfaltigkeit, d. h. das Schnittgebilde unserer eigentlichen A-R-H mit einer Ebene $D\left(u^{1}\right)$. Ich behaupte: Jede Breitenmannigfaltigkeit einer eigentlichen $A-R-H$ ist eine eigentliche $(n-1)$-dimensionale Affuhypersphäre, deren Affinmittelpunkt auf der Achse a liegt.

Bezeichnen wir die Grössen der Breitenmannigfaltigkeit durch Überstreichen, so ist

$$
\frac{1}{n-1} \bar{G}^{k l} \frac{\partial^{2} \underline{v}}{\partial u^{k} \partial u^{l}}=\frac{\bar{G}^{k l}}{n-1} \hat{\Gamma}_{k l}^{\prime} l_{r}+\bar{y}
$$




$$
\left.=\frac{\bar{G}^{k l}}{n-1}\left(A_{k l}^{m}+\Gamma_{k l}^{m}\right) \mathfrak{x}_{m}+\frac{\bar{G}_{i}^{k l}}{n-1} G_{k l} l\right) \quad(k, l, r>1, m \geqq 1)
$$

also

$$
\left.\overline{\mathfrak{n}}=\frac{\overline{G_{i}^{n l l}}}{n-1}\left[\left(A_{k l}^{1}+\Gamma_{k l}^{1}\right) \mathfrak{x}_{1}+G_{k l} \mathfrak{l}\right)\right]
$$

Nun sei $S$ der Schnittpunkt der Achse a mit einer Ebene $D\left(u^{1}\right)$. Wegen

$$
\left.\frac{\partial^{2} \underline{r}}{\partial u^{k} \partial u^{b}}=\left(A_{k l}^{m}+\Gamma_{k l}^{m}\right) \mathfrak{x}_{m}+G_{k l}\right)
$$

fällt die Komponente

$$
\mathfrak{v}_{(k l)}=\left(A_{k l l}^{1}+\Gamma_{k l}^{1}\right) \underline{x}_{1}+G_{k l l}^{1} \mathrm{r}
$$

des Vektors $\frac{\partial^{2} x}{\partial u^{k}} \frac{\partial u^{l}}{\text { }}$, welche in der Ebene $\eta_{1}$ liegt, in die Schnittgerade von $\eta_{1}$ und $D\left(u^{1}\right)$, welche den Punkt $S$ enthält. Dann aber geht nach (14) die Affinnormale der Breitenmannigfaltigkeit auch durch $S$; da aber der Punkt $S$ für alle Punkte einer und derselben Breitenmannigfaltigkeit derselbe ist, so ist jede Breitenmannigfaltigkeit eine eigentliche Affinhypersphäre, deren Affinmittelpunkt $S$ auf der Achse a liegt, w.z.b.w.

Nun bleibt nur noch eine Eigenschaft dieser Breitenaffinhypersphären zu beweisen. Nach $\left(3^{a}\right)$ sind je zwei entsprechende Kurven in zwei (parallelen) Ebenen $\mathfrak{D}\left(u^{1}\right)$ und $\mathfrak{D}\left(u_{0}^{1}\right)$ desselben Meridians gleichgerichtet, und dies für jede Richtung in den Breitenmannigfaltigkeiten. Daraus folgt aber, dass je zwei Breitenaffinhypersphären einander ähnlich und bezüglich der Achse a zueinander ähnlich gelegen sind. Hiermit sind im Falle eigentlicher A-R-H alle in $\mathrm{Nr} .2$ genannten Behauptungen bewiesen.

\section{Uneigentliche A-R-H.}

8. Wir führen bei uneigentlichen $A-R-H$ ausser den Vektoren $j^{(i)}$ in $\left(1^{\prime}\right)$ noch zwei die zweidimensionale Ebene $\eta$ aufspannende Vektoren $a$ und $b$ durch die Gleichungen ein:

$$
a^{2}=b^{2}=1 ; a_{3}^{(i)}=b_{3}^{(i)}=a b=0 \quad(i=1,2, \ldots \ldots, n-1) .
$$

Die Schnittkurven der A-R-H mit den zu $\eta$ parallelen Ebenen sind die Meridiane, die dann wieder zugleich Affinkrümmungslinien sind. Wir wählen sie zu $u^{1}-$ Kurven. Eine Breitenmannigfaltigkeit ist dadurch 
gekennzeichnet, dass läng's ihr die Affinnomalen einander parallel sind, also für jede ihrer Kurven $\left(u^{h}\right)^{1}(k>1)$

$$
\mathfrak{y} \times \mathfrak{t}_{k}=0
$$

d. h. wegen $G \neq 0$

$$
\mathfrak{y}_{k}=\frac{1}{R_{x_{i}}}=0 \quad(k>1)
$$

ist. Indem wir uneigentliche Affinhypersphären beiseite lassen, nehmen wir an, dass $\frac{1}{R_{1}} \neq 0$ ist; dann ist auch $\mathfrak{y}_{1} \neq 0$. Bei der Wahl eines konjugierten. Parameterkurvennetzes ist

$$
G_{i k}=0 \quad(i \neq k),
$$

also $X_{k}$ auf der Ebene $r$, die auch ron $r_{1}$ und $r$ aufgespannt wird, senkrecht. Daraus folgt wieder

$$
\mathfrak{X}_{k} \times \frac{\partial \mathfrak{x}_{k}}{\partial u^{1}}=0
$$

und dieselbe Gleichung für irgend eine der Breitenmannigfaltigkeit angehörende Kurve. Man schliesst dann wie bei $\left(13^{\circ}\right)$, dass

$$
-A_{1 k}^{k^{\prime}}+\Gamma_{1 k}^{k^{\prime}}=-A_{1 l}^{l^{\prime}}+\Gamma_{1 l}^{l^{\prime}} \quad(k, l>1)
$$

ist. Da ferner $x_{l}$ stets der Ebene $\eta$ angehört, so ist wegen

$$
\begin{gathered}
\left.\frac{\partial \mathfrak{x}_{1}}{\partial u^{i}}=\left(A_{1 i}^{s}+\Gamma_{1 i}^{s}\right) x_{s}+G_{1 i} i\right): \\
\left\{\begin{array}{l}
A_{1 k}^{b}+\Gamma_{1 i}^{l}=0 \\
A^{k}{ }_{11}+\Gamma^{k}{ }_{11}=0
\end{array}\right\}(k, l>1),
\end{gathered}
$$

nach $\left(6^{\prime}\right)$ also

$$
A_{1 k}^{k^{\prime}}=A_{1 l}^{l^{\prime}}, \Gamma_{1 k}^{k^{\prime}}=\Gamma_{1 l}^{z^{\prime}} \quad\left(k=k^{\prime}>1, l=l^{\prime}>1\right) .
$$

Da nun nach unserer Definition der $A-P \cdot H$ nach (3)

$$
A_{1 k}^{i}+\Gamma^{i}{ }_{1 i}=0 \quad(k>1, i \geq 1, i \neq k)
$$

ist, so folgt wieder wie in (9) und (10) nash $\left(7^{\prime}\right)$

$$
\begin{aligned}
& A_{11}=\Gamma_{11, k}=\frac{\partial G_{11}}{\partial u^{k}}=0 \quad(k>1), \\
& G_{11}= \pm 1, \quad \Gamma_{11}^{1}=0 .
\end{aligned}
$$


Da auch $\frac{\partial^{3} \mathfrak{X}}{\left(\partial^{1}\right)^{2} \partial u^{k}}$ auf $\eta$ senkrecht stehen muss $(k>1)$, so folgt wie (12)

$$
\frac{\partial A_{11}^{1}}{\partial u^{k}}=0 \quad(k>1) .
$$

Nach $\left(\boldsymbol{t}^{\prime}\right),\left(8^{\prime}\right),\left(10^{\prime}\right)$ und den Apolaritätsbeziehungen ist num.

$$
\frac{\partial}{\partial u^{k}}\left(A_{s k}^{k^{\prime}}\right)=\frac{\partial}{\partial u^{k}}\left(\Gamma_{1_{i}}^{k^{\prime}}\right)=0
$$

und wie in II verschwinden die Determinanten $\Delta_{1}$ und $\Delta_{2}$.

Die Breitenmannigfaltigkeiten liegen wieder in n-dimensionalen Ebenen $D\left(u^{1}\right)$, die nach $\left(3^{a}\right)$ wieder einander parallel sind. Wie in Nr. 7 zeigt man, dass die Affinnormalen einer Breitenmannigfaltigkeit alle einander parallel sind. Nach Nr. 4 sind also die Breitenmannigfaltigkeiten einander ähnliche und ähnlich gelegene uneigentliche $(n-1)$ - dimensionale Affinhypersphären, w. z. b. w. 